\title{
Multiparameter Optimization in Directed Evolution:
}

\section{Engineering Thermostability, Enantioselectivity, and Activity of an Epoxide Hydrolase}

Guangyue Li1,2,\$, Hui Zhang ${ }^{3, \S}$,Zhoutong Sun ${ }^{1,2}$,Xinqi Liu ${ }^{3, *}$ and Manfred T. Reetz ${ }^{1,2, *}$

${ }^{1}$ Max-Planck-Institut für Kohlenforschung, Kaiser-Wilhelm-Platz 1, 45470 Mülheim an der Ruhr, Germany

${ }^{2}$ Fachbereich Chemie der Philipps-Universität, Hans-Meerwein-Strasse, 35032 Marburg, Germany

35tate Key Laboratory of Medicinal Chemical Biology, College of Life Sciences, Nankai University, 300071 Tianjin, People's Republic of China

\section{Contents}

Supplementary Figures and Tables 3

Figure S1. Primer design and creation of LEH mutant LEH-P. 3

Figure S2. Primer design and creation of LEH mutant LEH-F1b. 3

Figure S3. Primer design and creation of LEH mutant SS-8. 3

Figure $\mathrm{S}_{4}$. Primer design and creation of LEH mutant RR-8. 3

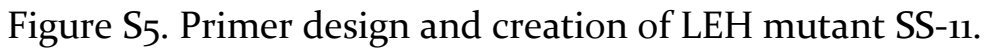
4

Figure S6. Primer design and creation of LEH mutant RR-11. 4

Figure $\mathrm{S}_{7}$. Primer design and creation of LEH mutant $\mathrm{H}-2-\mathrm{H}_{5}-1$ using $\mathrm{H}_{-2}-\mathrm{H}_{5}$ as template

Figure S8. Primer design and library creation of LEH for library A (red) and B (green). Valine $(\mathrm{V})$, phenylalanine $(\mathrm{F})$ and tyrosine $(\mathrm{Y})$ were used as the building blocks for residues L74, M78, L114 and I116; Lysine (K), Proline (P) and Aspartic acid (D) were used as the building blocks for residues $\mathrm{S}_{15}, \mathrm{~A}_{19}, \mathrm{~T}_{7} 6$ and T85 .........5

Figure S9. Primer design and library creation of LEH for library C (blue) and D (purple). Valine $(\mathrm{V})$, phenylalanine $(\mathrm{F})$ and tyrosine $(\mathrm{Y})$ were used as the building blocks for residues S32, I8o, F139 and L147; Lysine (K), Proline (P) and Aspartic acid (D) were used as the building blocks for residues E45, E124, N92 andY96.......5 
Figure S1o. SDS-PAGE for $\mathrm{H}-2-\mathrm{H}_{5}$ after purification

Table S1. The information of site-directed mutants.

Table S2. Results of screening libraries A, B, C and D of LEH for substrate (1) using a tripe code (V-F-Y for stereoselectivity related sites and K-P-D for thermostability related sites).

.6

Table S3. The stereoselectivity of LEH variants $\mathrm{H}-2-\mathrm{H}_{5}$ and $\mathrm{J}-7-\mathrm{A}_{2}$ as catalysts in the model reaction (Scheme 1 ) at $37^{\circ} \mathrm{C}$.

Table S4. Data collection and refinement statistics............................................ 8

Table S5. List of primers for site-directed mutagenesis of LEH..............................10

Table S6. List of primers for LEH libraries A, B, C and D.......................................12

Table S7. List of primers for ISM of LEH...........................................................

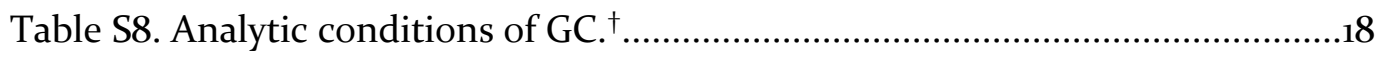




\section{Supplementary Figures and Tables}

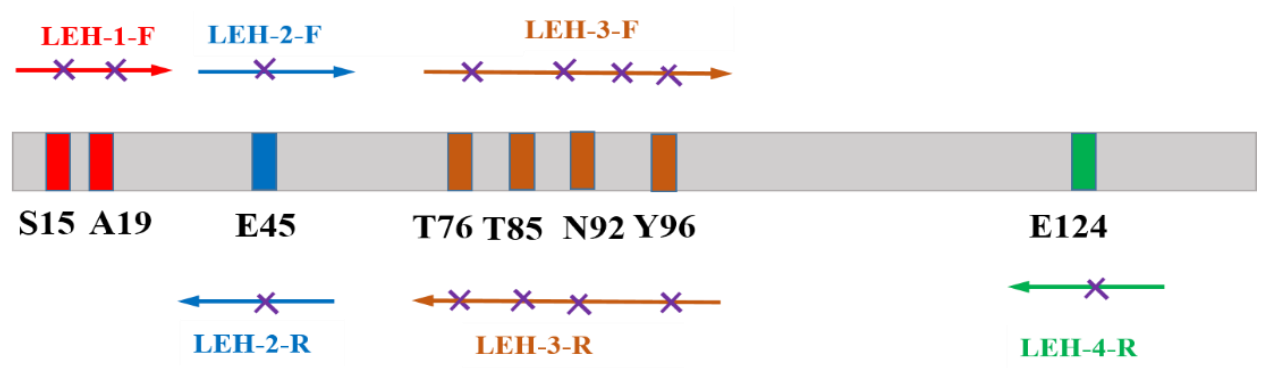

Figure S1. Primer design and creation of LEH mutant LEH-P.

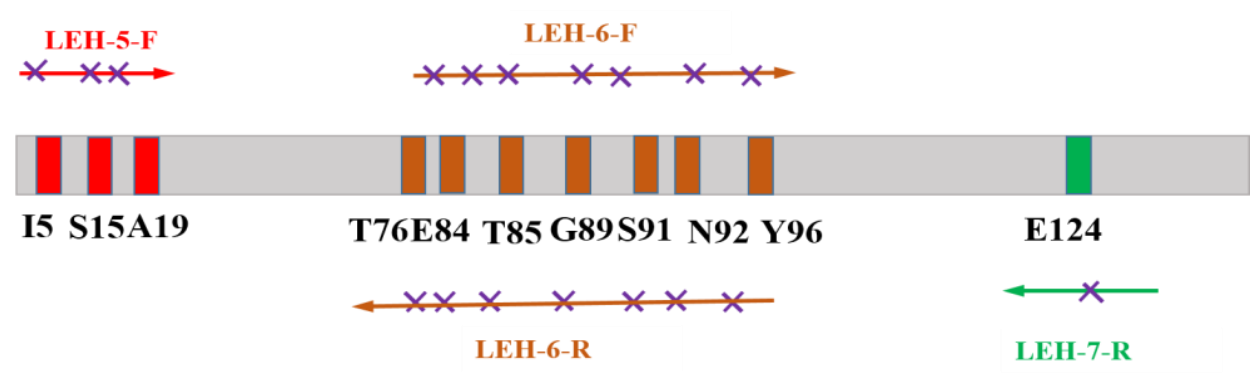

Figure S2. Primer design and creation of LEH mutant LEH-Fıb.

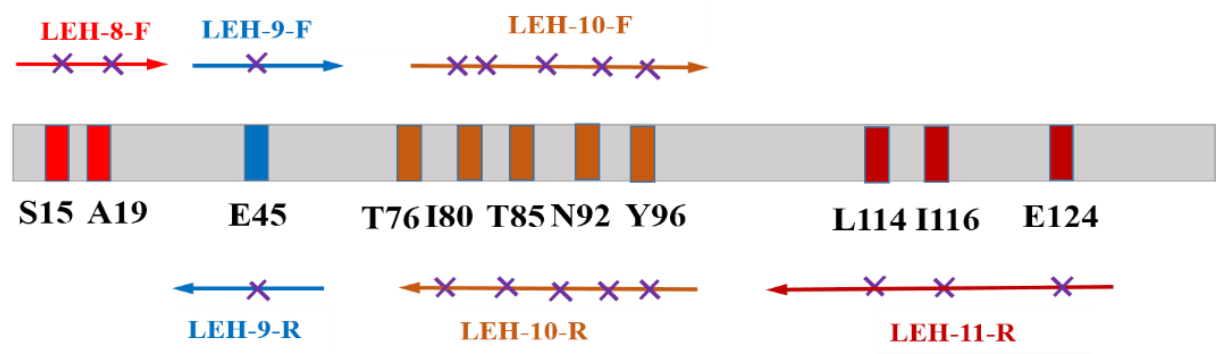

Figure S3. Primer design and creation of LEH mutant SS-8.

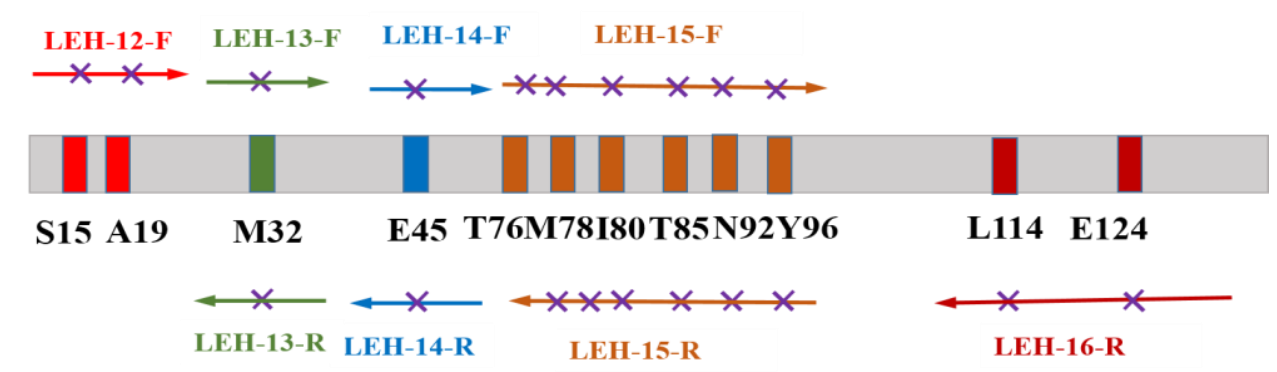

Figure $\mathbf{S}$. Primer design and creation of LEH mutant RR-8. 


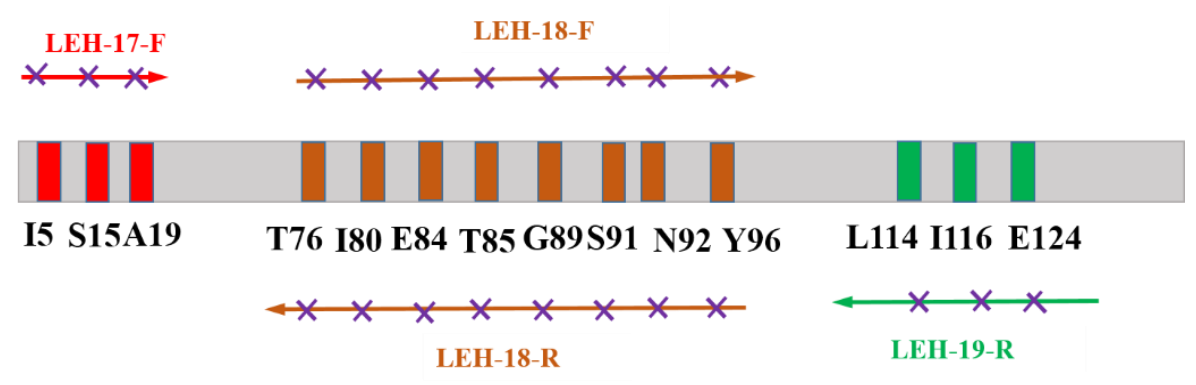

Figure S5. Primer design and creation of LEH mutant SS-11.

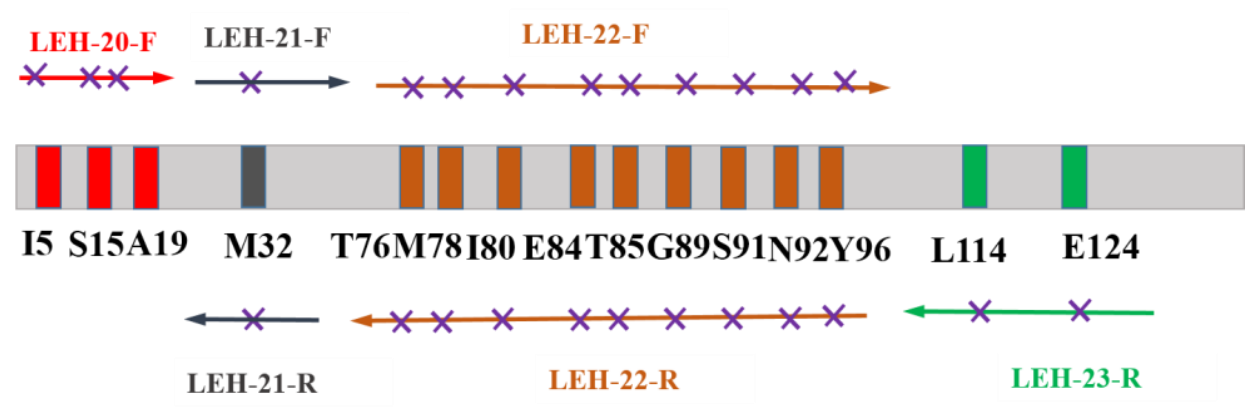

Figure S6. Primer design and creation of LEH mutant RR-11.

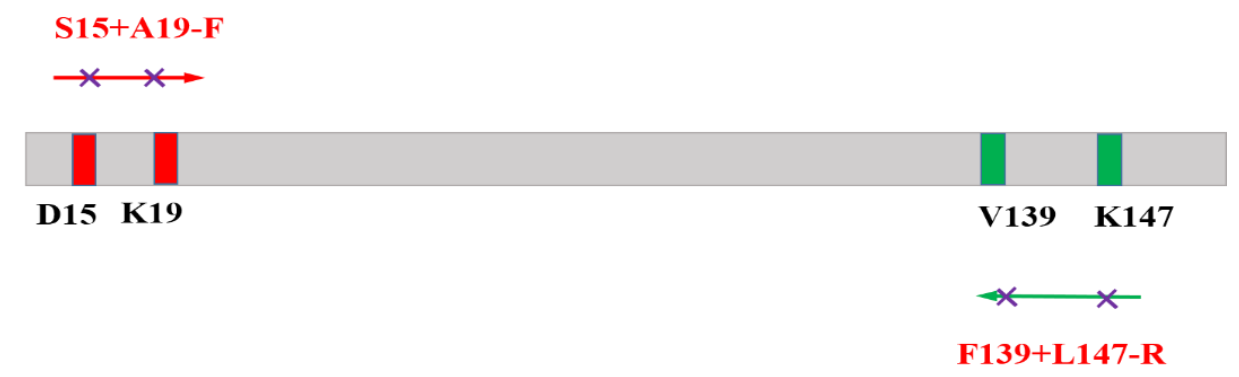

Figure $\mathrm{S}_{7}$. Primer design and creation of LEH mutant $\mathrm{H}_{-2}-\mathrm{H}_{5}-1$ using $\mathrm{H}-2-\mathrm{H}_{5}$ as template 


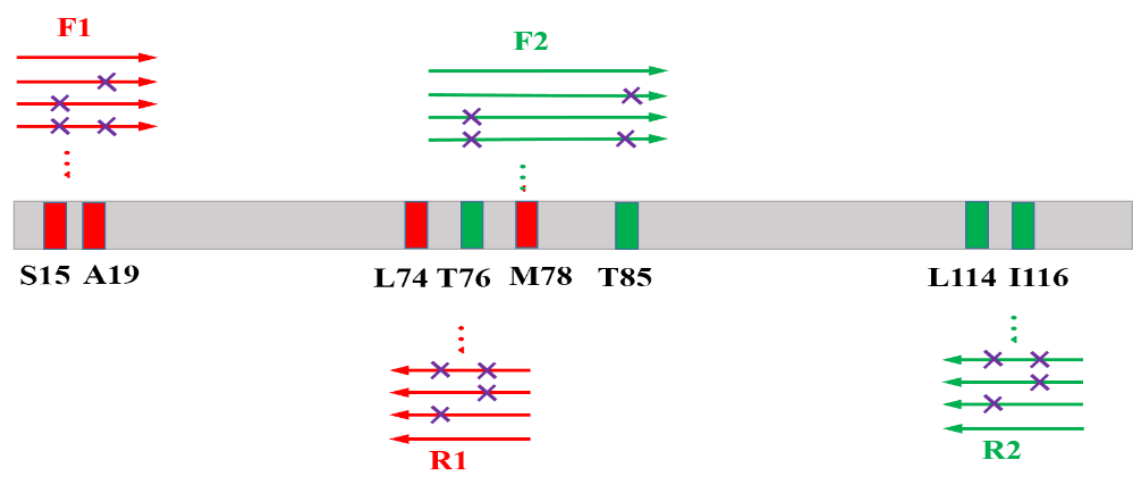

Figure S8. Primer design and library creation of LEH for library A (red) and B (green). Valine $(\mathrm{V})$, phenylalanine $(\mathrm{F})$ and tyrosine $(\mathrm{Y})$ were used as the building blocks for residues L74, M78, L114 and In6; Lysine (K), Proline (P) and Aspartic acid (D) were used as the building blocks for residues $\mathrm{S}_{15}, \mathrm{~A}_{19}, \mathrm{~T}_{7} 6$ and $\mathrm{T} 85$.

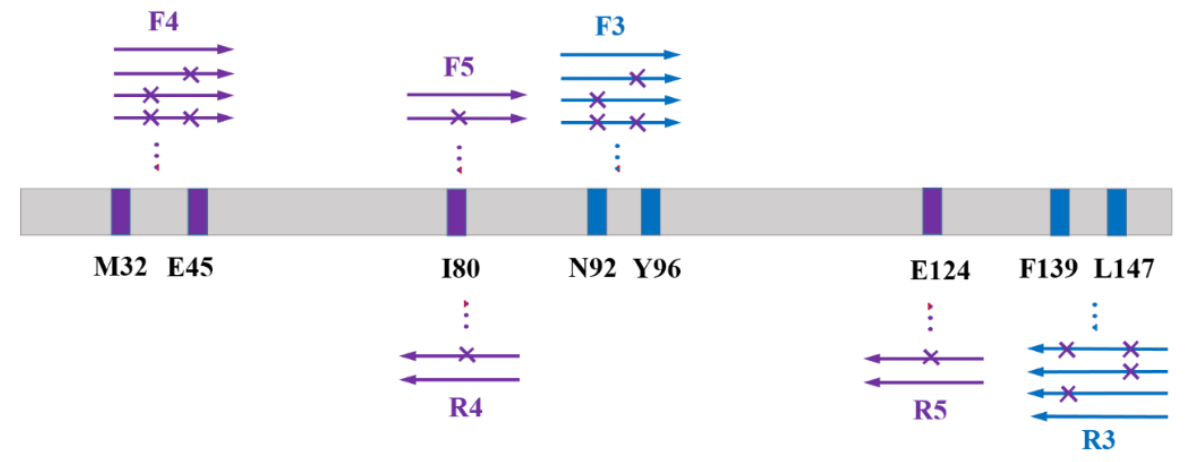

Figure S9. Primer design and library creation of LEH for library C (blue) and D (purple). Valine $(\mathrm{V})$, phenylalanine $(\mathrm{F})$ and tyrosine $(\mathrm{Y})$ were used as the building blocks for residues S32, I8o, F139 and L147; Lysine (K), Proline (P) and Aspartic acid (D) were used as the building blocks for residues E45, E124, N92 andY96.

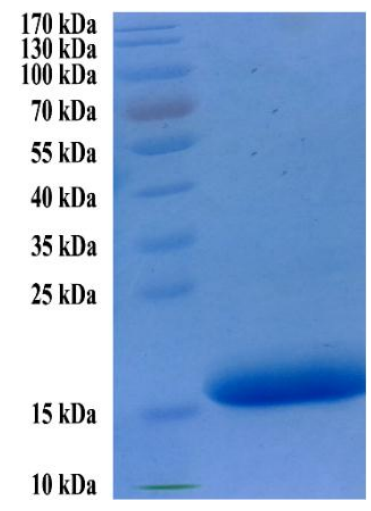

Figure S1o. SDS-PAGE for $\mathrm{H}_{-2}-\mathrm{H}_{5}$ after purification 
Table S1. The information of site-directed mutants.

\begin{tabular}{|c|c|}
\hline Mutants & Mutations \\
\hline LEH-P & $\mathrm{S}_{15} \mathrm{P} / \mathrm{A}_{19} \mathrm{~K} / \mathrm{E}_{45} \mathrm{~K} / \mathrm{T}_{7} 6 \mathrm{~K} / \mathrm{T}_{85} \mathrm{~V} / \mathrm{N}_{92} \mathrm{~K} / \mathrm{Y}_{96} 6 \mathrm{~F} / \mathrm{E}_{124} \mathrm{D}$ \\
\hline LEH-F1b & $\begin{array}{l}\mathrm{I}_{5} \mathrm{C} / \mathrm{S}_{15} \mathrm{P} / \mathrm{A}_{19} \mathrm{~K} / \mathrm{T}_{7} 6 \mathrm{~K} / \mathrm{E} 84 \mathrm{C} / \mathrm{T} 85 \mathrm{~V} / \mathrm{G}_{89} \mathrm{C} / \mathrm{S}_{91} \mathrm{C} / \mathrm{N}_{92} \mathrm{~K} / \mathrm{Y} 96 \mathrm{~F} / \\
\mathrm{E}_{124} \mathrm{D}\end{array}$ \\
\hline $\mathrm{SZ}_{348}$ & I8oY/ L114V/ I116V \\
\hline $\mathrm{SZ}_{529}$ & 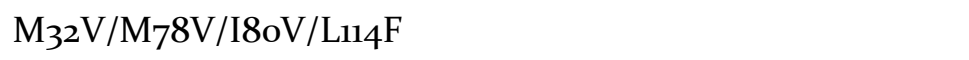 \\
\hline SS-8 & $\begin{array}{l}\mathrm{S}_{15} \mathrm{P} / \mathrm{A}_{19} \mathrm{~K} / \mathrm{E}_{45} \mathrm{~K} / \mathrm{T} 76 \mathrm{~K} / \mathrm{T} 85 \mathrm{~V} / \mathrm{N}_{92} \mathrm{~K} / \mathrm{Y}_{96} 6 \mathrm{~F} / \mathrm{E}_{124} \mathrm{D} / \\
\mathrm{I} 8 \mathrm{oY} / \mathrm{L}_{114} \mathrm{~V} / \mathrm{I} 116 \mathrm{~V}\end{array}$ \\
\hline RR-8 & $\begin{array}{l}\mathrm{S}_{15} \mathrm{P} / \mathrm{A}_{19} \mathrm{~K} / \mathrm{M}_{32} \mathrm{~V} / \mathrm{E}_{45} \mathrm{~K} / \mathrm{T}_{7} 6 \mathrm{~K} / \mathrm{M}_{78} \mathrm{~V} / \mathrm{I} 80 \mathrm{~V} / \mathrm{T}_{85} \mathrm{~V} / \mathrm{N}_{92} \mathrm{~K} / \mathrm{Y}_{96} \\
\mathrm{~F} / \mathrm{L}_{14} \mathrm{~F} / \mathrm{E}_{124} \mathrm{D}\end{array}$ \\
\hline SS-11 & $\begin{array}{l}\mathrm{I}_{5} \mathrm{C} / \mathrm{S}_{15} \mathrm{P} / \mathrm{A}_{19} \mathrm{~K} / \mathrm{T}_{7} 6 \mathrm{~K} / \mathrm{I} 8 \mathrm{oY} / \mathrm{E} 84 \mathrm{C} / \mathrm{T} 85 \mathrm{~V} / \mathrm{G} 89 \mathrm{C} / \mathrm{S}_{91} \mathrm{C} / \mathrm{N}_{92} \mathrm{~K} / \mathrm{Y} \\
96 \mathrm{~F} / \mathrm{L}_{11} \mathrm{~V} / \mathrm{I} 116 \mathrm{~V} / \mathrm{E}_{124} \mathrm{D}\end{array}$ \\
\hline RR-11 & $\begin{array}{l}\mathrm{I}_{5} \mathrm{C} / \mathrm{S}_{15} \mathrm{P} / \mathrm{A}_{19} \mathrm{~K} / \mathrm{M}_{32} \mathrm{~V} / \mathrm{T}_{7} 6 \mathrm{~K} / \mathrm{M}_{78} \mathrm{~V} / \mathrm{I} 8 \mathrm{oV} / \mathrm{E} 84 \mathrm{C} / \mathrm{T}_{55} \mathrm{~V} / \mathrm{G} 89 \mathrm{C} \\
/ \mathrm{S}_{91} \mathrm{C} / \mathrm{N}_{92} \mathrm{~K} / \mathrm{Y}_{96} 6 \mathrm{~F} / \mathrm{L}_{114} \mathrm{~F} / \mathrm{E}_{124} \mathrm{D}\end{array}$ \\
\hline
\end{tabular}

Table S2. Results of screening libraries A, B, C and D of LEH for substrate (1) using a tripe code (V-F-Y for stereoselectivity related sites and K-P-D for thermostability related sites).

\begin{tabular}{|c|c|c|c|c|c|c|}
\hline Library & Code & Mutation & ee\% & $\mathrm{C} \%$ & $\begin{array}{c}\text { Favored } \\
\text { enantiome } \\
r\end{array}$ & $\begin{array}{l}\text { Thermos } \\
\text { tability }\end{array}$ \\
\hline template & WT & & 2 & 100 & $(S, S)$ & - \\
\hline \multirow{5}{*}{ A } & $\mathrm{A}-1-\mathrm{B}_{4}$ & $\mathrm{~S}_{15} \mathrm{D} / \mathrm{L}_{74} \mathrm{~F} / \mathrm{M}_{7} 8 \mathrm{Y}$ & 44 & 97 & $(S, S)$ & - \\
\hline & $\mathrm{A}-1-\mathrm{H}_{4}$ & $\mathrm{M}_{78 \mathrm{Y}}$ & 34 & 96 & $(R, R)$ & -- \\
\hline & $A-5-C_{1}$ & $\mathrm{~S}_{15} \mathrm{P} / \mathrm{M}_{7} 8 \mathrm{~F}$ & 34 & 100 & $(R, R)$ & ++ \\
\hline & A-7-A11 & $\mathrm{S}_{15} \mathrm{D} / \mathrm{A}_{19} \mathrm{D} / \mathrm{L}_{74} \mathrm{~F}$ & 42 & 95 & $(S, S)$ & - \\
\hline & A-8-D5 & $\mathrm{A}_{19} \mathrm{~K} / \mathrm{L}_{74} \mathrm{~V} / \mathrm{M}_{78} \mathrm{~F}$ & 34 & 99 & $(R, R)$ & - \\
\hline
\end{tabular}




\begin{tabular}{|c|c|c|c|c|c|c|}
\hline Library & Code & Mutation & ee\% & $\mathrm{C} \%$ & $\begin{array}{c}\text { Favored } \\
\text { enantiome } \\
r\end{array}$ & $\begin{array}{l}\text { Thermos } \\
\text { tability }\end{array}$ \\
\hline template & WT & & 2 & 100 & $(S, S)$ & - \\
\hline \multirow{3}{*}{ B } & $\mathrm{B}-1-\mathrm{F} 12$ & $\mathrm{~T}_{76} \mathrm{~K} / \mathrm{L} 114 \mathrm{~V} / \mathrm{In} 6 \mathrm{~V}$ & 71 & 95 & $(S, S)$ & + \\
\hline & B-4-B11 & $\mathrm{T} 76 \mathrm{D} / \mathrm{L} 114 \mathrm{~V} / \mathrm{I} 116 \mathrm{~V}$ & 71 & 91 & $(S, S)$ & - \\
\hline & B-6-Gio & $\mathrm{T}_{7} 6 \mathrm{~K} / \mathrm{T} 8{ }_{5} \mathrm{P} / \mathrm{I} 116 \mathrm{~V}$ & 55 & 95 & $(S, S)$ & + \\
\hline template & WT & & 2 & 100 & $(S, S)$ & - \\
\hline C & ND & & & & & \\
\hline template & WT & & 2 & 100 & $(S, S)$ & - \\
\hline D & ND & & & & & \\
\hline template & $A-5-C_{1}$ & $\mathrm{~S}_{15} \mathrm{P} / \mathrm{M}_{78 \mathrm{~F}}$ & 34 & 100 & $(R, R)$ & ++ \\
\hline \multirow{2}{*}{$A \rightarrow C$} & E-5-A8 & $\mathrm{S}_{15} \mathrm{P} / \mathrm{M} 78 \mathrm{~F} / \mathrm{N}_{92} \mathrm{~K} / \mathrm{F}_{139} \mathrm{~V}$ & 39 & 100 & $(R, R)$ & ++ \\
\hline & $\mathrm{E}-5-\mathrm{A}_{3}$ & $\mathrm{~S}_{15} \mathrm{P} / \mathrm{M}_{7} 8 \mathrm{~F} / \mathrm{N}_{92} \mathrm{D} / \mathrm{F}_{139} \mathrm{~V}$ & 39 & 100 & $(R, R)$ & + \\
\hline template & E-5-A8 & $\mathrm{S}_{15} \mathrm{P} / \mathrm{M}_{7} 8 \mathrm{~F} / \mathrm{N}_{92} \mathrm{~K} / \mathrm{F}_{139} \mathrm{~V}$ & 39 & 100 & $(R, R)$ & ++ \\
\hline \multirow{3}{*}{$\mathrm{A}-\mathrm{C} \rightarrow \mathrm{B}$} & $\mathrm{I}-2-\mathrm{D}_{5}$ & $\begin{array}{c}\mathrm{S}_{15} \mathrm{P} / \mathrm{M}_{7} 8 \mathrm{~F} / \mathrm{N}_{92} \mathrm{~K} / \mathrm{F}_{139} \mathrm{~V} / \mathrm{T}_{7} \\
6 \mathrm{D} / \mathrm{T}_{8} \mathrm{~K}\end{array}$ & 43 & 90 & $(R, R)$ & - \\
\hline & $\mathrm{I}-4-\mathrm{H}_{10}$ & $\begin{array}{c}\mathrm{S}_{15} \mathrm{P} / \mathrm{M}_{7} 8 \mathrm{~F} / \mathrm{N}_{92} \mathrm{~K} / \mathrm{F}_{139} \mathrm{~V} / \mathrm{T}_{7} \\
6 \mathrm{~K} / \mathrm{T}_{85} \mathrm{~K}\end{array}$ & 44 & 100 & $(R, R)$ & + \\
\hline & I-5-E11 & $\begin{array}{c}\mathrm{S}_{15} \mathrm{P} / \mathrm{M}_{78} 8 \mathrm{~F} / \mathrm{N}_{92} \mathrm{~K} / \mathrm{F}_{139} \mathrm{~V} / \mathrm{T} 8 \\
{ }_{5} \mathrm{~K}\end{array}$ & 44 & 100 & $(R, R)$ & - \\
\hline template & $\mathrm{I}-4-\mathrm{H}_{10}$ & $\begin{array}{c}\mathrm{S}_{15} \mathrm{P} / \mathrm{M}_{7} 8 \mathrm{~F} / \mathrm{N}_{92} \mathrm{~K} / \mathrm{F}_{139} \mathrm{~V} / \mathrm{T}_{7} \\
6 \mathrm{~K} / \mathrm{T}_{85} \mathrm{~K}\end{array}$ & 44 & 100 & $(R, R)$ & + \\
\hline$A-C-B \rightarrow D$ & $\mathrm{~J}-7-\mathrm{A} 12$ & $\begin{array}{l}\mathrm{S}_{15} \mathrm{P} / \mathrm{M}_{78} \mathrm{~F} / \mathrm{N}_{92} \mathrm{~K} / \mathrm{F}_{139} \mathrm{~V} / \mathrm{T}_{7} \\
6 \mathrm{~K} / \mathrm{T}_{5} \mathrm{~K} / \mathrm{E}_{45} \mathrm{D} / \mathrm{I} 8 \mathrm{oV} / \mathrm{E}_{124} \mathrm{D}\end{array}$ & 80 & 100 & $(R, R)$ & ++ \\
\hline template & B-1-F12 & T76K/L114V/In6V & 71 & 95 & $(S, S)$ & + \\
\hline
\end{tabular}




\begin{tabular}{|c|c|c|c|c|c|c|}
\hline Library & Code & Mutation & ee\% & $\mathrm{C} \%$ & $\begin{array}{c}\text { Favored } \\
\text { enantiome } \\
r\end{array}$ & $\begin{array}{l}\text { Thermos } \\
\text { tability }\end{array}$ \\
\hline \multirow{3}{*}{$B \rightarrow C$} & F-1-E4 & $\begin{array}{c}\mathrm{T}_{76 \mathrm{~K} / \mathrm{L} 114 \mathrm{~V} / \mathrm{I}_{116 \mathrm{~V}} / \mathrm{N}_{92} \mathrm{D} / \mathrm{F} 1} \\
39 \mathrm{~V} / \mathrm{L}_{47} \mathrm{~F}\end{array}$ & 82 & 95 & $(S, S)$ & + \\
\hline & F-2-H1o & $\begin{array}{c}\mathrm{T}_{76 \mathrm{~K} / \mathrm{L} 114 \mathrm{~V} / \mathrm{I} 116 \mathrm{~V}} \\
/ \mathrm{F}_{39} \mathrm{~V} / \mathrm{L} 147 \mathrm{~F}\end{array}$ & 82 & 95 & $(S, S)$ & + \\
\hline & F-6-A9 & $\begin{array}{c}\mathrm{T}_{76 \mathrm{~K}} / \mathrm{L}_{114} \mathrm{~V} / \mathrm{I}_{116 \mathrm{~V}} / \mathrm{N}_{92} \mathrm{~K} / \mathrm{F}_{13} \\
\text { 9V/L147F }\end{array}$ & 83 & 99 & $(S, S)$ & ++ \\
\hline template & F-6-A9 & $\begin{array}{c}\mathrm{T}_{76 \mathrm{~K}} / \mathrm{L}_{114} \mathrm{~V} / \mathrm{I}_{116 \mathrm{~V}} / \mathrm{N}_{92} \mathrm{~K} / \mathrm{F}_{13} \\
\text { 9V/L147F }\end{array}$ & 83 & 99 & $(S, S)$ & ++ \\
\hline \multirow{3}{*}{$\mathrm{B}-\mathrm{C} \rightarrow \mathrm{A}$} & G-3-A8 & $\begin{array}{l}\mathrm{T}_{76 \mathrm{~K} / \mathrm{L} 114 \mathrm{~V} / \mathrm{I}_{116} 6 \mathrm{~V} / \mathrm{N}_{92} \mathrm{~K} / \mathrm{F}_{13}} \\
9 \mathrm{~V} / \mathrm{L}_{47} \mathrm{~F} / \mathrm{S}_{15} \mathrm{D} / \mathrm{L}_{74} \mathrm{~F} / \mathrm{M}_{78 \mathrm{~F}}\end{array}$ & 92 & 100 & $(S, S)$ & ++ \\
\hline & G-6-E6 & 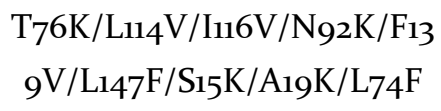 & 90 & 90 & $(S, S)$ & ++ \\
\hline & $\mathrm{G}-7-\mathrm{H}_{2}$ & $\begin{array}{c}\mathrm{T}_{76 \mathrm{~K} / \mathrm{L}_{11} \mathrm{~V} / \mathrm{I}_{116} 6 \mathrm{~V} / \mathrm{N}_{92} \mathrm{~K} / \mathrm{F}_{13}} \\
9 \mathrm{~V} / \mathrm{L} 147 \mathrm{~F} / \mathrm{S}_{15} \mathrm{D} / \mathrm{A}_{19} \mathrm{~K} / \mathrm{L}_{74} \mathrm{~F} / \\
\mathrm{M}_{78 \mathrm{~F}}\end{array}$ & 92 & 100 & $(S, S)$ & +++ \\
\hline template & $\mathrm{G}-7-\mathrm{H}_{2}$ & 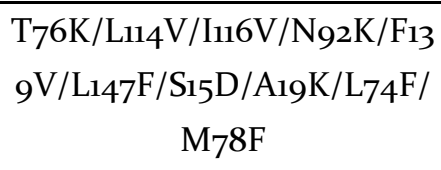 & 92 & 100 & $(S, S)$ & +++ \\
\hline B-C-A $\rightarrow$ D & $\mathrm{H}-2-\mathrm{H}_{5}$ & 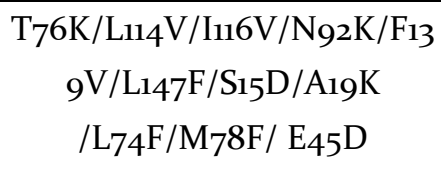 & 94 & 100 & $(S, S)$ & ++ \\
\hline
\end{tabular}

ND: not detected; -: Thermostability is similar or lower WT LEH; +, ++, +++: Thermostability is higher than WT LEH and increase gradually.

Table S3. The stereoselectivity of LEH variants $\mathrm{H}-2-\mathrm{H}_{5}$ and J-7-A12 as catalysts in the model reaction (Scheme 1 ) at $37^{\circ} \mathrm{C}$.

\begin{tabular}{|l|l|l|l|l|}
\hline Entry & Enzyme & ee (\%) & $\begin{array}{l}\text { Preferred } \\
\text { enantiomer }\end{array}$ & Mutations \\
\hline 1 & WT LEH & 2 & $(S, S)$ & \\
\hline 2 & $\mathrm{H}-2-\mathrm{H}_{5}$ & 92 & $(S, S)$ & $\begin{array}{l}\text { T76K/L114V/I116V/N92K/F139V/L147F/S } \\
15 \mathrm{D} / \mathrm{L}_{74} \mathrm{~F} / \mathrm{M}_{7} 8 \mathrm{~F} / \mathrm{E}_{45} \mathrm{D}\end{array}$ \\
\hline 3 & $\mathrm{~J}-7-\mathrm{A} 12$ & 79 & $(R, R)$ & $\begin{array}{l}\mathrm{S} 15 \mathrm{P} / \mathrm{M} 78 \mathrm{~F} / \mathrm{N}_{92} \mathrm{~K} / \mathrm{F}_{139} \mathrm{~V} / \mathrm{T} 76 \mathrm{~K} / \mathrm{T} 85 \mathrm{~K} / \mathrm{E} \\
45 / \mathrm{D} / 8 \mathrm{OV} / \mathrm{E}_{124} \mathrm{D}\end{array}$ \\
\hline
\end{tabular}


Table $\mathbf{S}_{4}$. Data collection and refinement statistics.

\begin{tabular}{|c|c|c|}
\hline Name & $\mathrm{H}-2-\mathrm{H}_{5}$ & $\begin{array}{c}\text { H-2-H5- }(S, S) \text {-cyclohexane- } \\
\text { 1,2-diol complex }\end{array}$ \\
\hline PDB ID & $5 \mathrm{JPP}$ & 5JPU \\
\hline \multicolumn{3}{|l|}{ Data collection } \\
\hline Beamline & SSRF-BLi7U & SSRF-BL17U \\
\hline Wavelength $(\AA)$ & 0.9792 & 0.9792 \\
\hline Space group & $P_{41}$ & $P_{41}$ \\
\hline Unit cell parameters $\left(\AA \AA^{\circ}{ }^{\circ}\right)$ & $\begin{array}{l}a=65.4 \\
b=65.4 \\
c=72.4 \\
\alpha=\beta=\gamma=90\end{array}$ & $\begin{array}{l}a=65.5 \\
b=65.5 \\
c=72.9 \\
\alpha=\beta=\gamma=90\end{array}$ \\
\hline Resolution $(\AA)^{\mathrm{a}}$ & $50-2.30(2.44-2.30)$ & $50-1.89(1.96-1.89)$ \\
\hline No of measured reflecions & 144183 & 733354 \\
\hline No of unique reflections $\mathrm{s}^{\mathrm{a}}$ & $11373(2264)$ & $25410(2464)$ \\
\hline Redoundancy $^{\mathrm{a}}$ & $5.5(6.3)$ & $14.8(15.0)$ \\
\hline Completeness $(\%)^{\mathrm{a}}$ & $84.0(100.0)$ & 100.0(100.0) \\
\hline Average $(\mathrm{I} / \sigma)^{\mathrm{a}}$ & $21.3(2.8)$ & $35.7(3.5)$ \\
\hline Rmerge $(\%)^{\mathrm{a}, \mathrm{b}}$ & $11.4(32.3)$ & $7.6(20.9)$ \\
\hline \multicolumn{3}{|l|}{ Refinement } \\
\hline No of reflections(work/free) & 10818(544) & $25357(1283)$ \\
\hline $\mathrm{R}_{\text {work }} / \mathrm{R}_{\text {free }}{ }^{\mathrm{c}}$ & $0.2671 / 0.2969$ & $0.2062 / 0.2248$ \\
\hline \multicolumn{3}{|l|}{ No of non-H atom } \\
\hline $\begin{array}{l}\text { Protein } \\
\text { ligand }\end{array}$ & $\begin{array}{l}1872 \\
0\end{array}$ & $\begin{array}{l}1872 \\
16\end{array}$ \\
\hline
\end{tabular}




\begin{tabular}{|l|l|l|}
\hline Name & H-2-H5 & $\begin{array}{l}\text { H-2-H5-(S,S)-cyclohexane- } \\
\text { 1,2-diol complex }\end{array}$ \\
\hline waters & 241 & 445 \\
\hline Average B factor[A $\left.{ }^{2}\right]$ & 26.53 & 17.05 \\
\hline Bond lengths(Å) & 0.007 & 0.007 \\
\hline Bond angles(०) & 1.139 & 1.160 \\
\hline $\begin{array}{l}\text { Ramachandran plot } \\
\text { favored(\%) }\end{array}$ & 100.00 & 99.14 \\
\hline $\begin{array}{l}\text { Ramachandran plot } \\
\text { allowed(\%) }\end{array}$ & 0.00 & 0.86 \\
\hline $\begin{array}{l}\text { Ramachandran plot } \\
\text { outlier(\%) }\end{array}$ & 0.00 & 0.00 \\
\hline
\end{tabular}

a Numbers in parentheses are values for the highest-resolution shell.

${ }^{\mathrm{b}} R$ merge $=\Sigma h k l \Sigma \mathrm{i}|I \mathrm{i}-\langle I\rangle| / \Sigma h k l \Sigma \mathrm{i}|\langle I\rangle|$, where Ii is the intensity for the $i$ th measurement of an equivalent reflection with indices $\mathrm{h}, \mathrm{k}$, and $\mathrm{l}$.

${ }^{c} R$ free was calculated with the $5 \%$ of reflections set aside randomly throughout the refinement.

Table S5. List of primers for site-directed mutagenesis of LEH.

\begin{tabular}{|c|c|}
\hline $\begin{array}{l}\text { Name of } \\
\text { primer }\end{array}$ & Sequence (5' to $\left.3^{\prime}\right)$ \\
\hline LEH-1-F & TGGGCGTCCAAGGACCCGGCCGCCGGCAAAGCCTCGACTCCGGACG \\
\hline LEH-2-F & TGATGCTGCAAAACTCATTAAATACTTTGCAGAAGACACGAT \\
\hline LEH-2-R & ATCGTGTCTTCTGCAAAGTATTTAATGAGTTTTGCAGCATCA \\
\hline LEH-3-F & $\begin{array}{l}\text { TGGCTGGCCTGTTCAAAGTCATGAGCATCGATGCGGTGGAGGTGTTCCATATCG } \\
\text { GCTCGAGTAAAGGACTTGTGTTTACCGAACGTGTCGA }\end{array}$ \\
\hline LEH-3-R & $\begin{array}{l}\text { TCGACACGTTCGGTAAACACAAGTCCTTTACTCGAGCCGATATGGAACACCTCCA } \\
\text { CCGCATCGATGCTCATGACTTTGAACAGGCCAGCCA }\end{array}$ \\
\hline LEH-4-R & CCCGTAATCTTGCCATCGGTGAGCTGGAAGACT \\
\hline LEH-5-F & $\begin{array}{l}\text { CACCACACATCAAAGTGCGAACAACCTCGCTGGGCGTCCAAGGACCCGGCCGCC } \\
\text { GGCAAAGCCTCGACTCCGGACGA }\end{array}$ \\
\hline
\end{tabular}




\begin{tabular}{|c|c|}
\hline $\begin{array}{l}\text { Name of } \\
\text { primer }\end{array}$ & Sequence ( 5 ' to $\left.3^{\prime}\right)$ \\
\hline LEH-6-F & $\begin{array}{l}\text { TGGCTGGCCTGTTCAAAGTCATGAGCATCGATGCGGTGTGCGTGTTCCATATCT } \\
\text { GCTCGTGCAAAGGACTTGTGTTTACCGAACGTGTCGA }\end{array}$ \\
\hline LEH-6-R & $\begin{array}{l}\text { TCGACACGTTCGGTAAACACAAGTCCTTTGCACGAGCAGATATGGAACACGCAC } \\
\text { ACCGCATCGATGCTCATGACTTTGAACAGGCCAGCCA }\end{array}$ \\
\hline LEH-7-R & Same as LEH-4-R \\
\hline LEH-8-F & Same as LEH-1-F \\
\hline LEH-9-F & Same as LEH-2-F \\
\hline LEH-9-R & Same as LEH-2-R \\
\hline LEH-10-F & $\begin{array}{l}\text { TGGCTGGCCTGTTCAAAGTCATGAGCTATGATGCGGTGGAGGTGTTCCATATCG } \\
\text { GCTCGAGTAAAGGACTTGTGTTTACCGAACGTGTCGA }\end{array}$ \\
\hline LEH-10-R & $\begin{array}{l}\text { TCGACACGTTCGGTAAACACAAGTCCTTTACTCGAGCCGATATGGAACACCTCCA } \\
\text { CCGCATCATAGCTCATGACTTTGAACAGGCCAGCCA }\end{array}$ \\
\hline LEH-11-R & $\begin{array}{l}\text { CCCGTAATCTTGCCATCGGTGAGCTGGAAGACTCCGAGCACTGACACGTTGTAG } \\
\text { СTCTTGC }\end{array}$ \\
\hline LEH-12-F & Same as LEH-1-F \\
\hline LEH-13-F & GATCGTTCTGGAGTTCGTGGACGCACTGACCAGTA \\
\hline LEH-13-R & TACTGGTCAGTGCGTCCACGAACTCCAGAACGATC \\
\hline LEH-14-F & Same as LEH-2-F \\
\hline LEH-14-R & Same as LEH-2-R \\
\hline LEH-15-F & $\begin{array}{l}\text { AAACTCTGGCTGGCCTTTTCAAAGTCGTGAGCGTGGATGCGGTGGAGGTGTTCC } \\
\text { ATATCGGCTCGAGTAAAGGACTTGTGTTTACCGAACGTGTCGA }\end{array}$ \\
\hline LEH-15-R & $\begin{array}{l}\text { TCGACACGTTCGGTAAACACAAGTCCTTTACTCGAGCCGATATGGAACACCTCCA } \\
\text { CCGCATCCACGCTCACGACTTTGAAAAGGCCAGCCAGAGTTT }\end{array}$ \\
\hline LEH-16-R & $\begin{array}{l}\text { CCCGTAATCTTGCCATCGGTGAGCTGGAAGACTCCGAGGATTGAAAAGTTGTAG } \\
\text { СТCTTGC }\end{array}$ \\
\hline LEH-17-F & Same as $\mathrm{LEH}-5-\mathrm{F}$ \\
\hline LEH-18-F & $\begin{array}{l}\text { GGCTGGCCTGTTCAAAGTCATGAGCTATGATGCGGTGTGCGTGTTCCATATCTG } \\
\text { CTCGTGCAAAGGACTTGTGTTTACCGAACGTGTCGA }\end{array}$ \\
\hline
\end{tabular}

$11 / 18$ 


\begin{tabular}{|l|l|}
\hline $\begin{array}{l}\text { Name of } \\
\text { primer }\end{array}$ & Sequence (5' to $\mathbf{3}$ ') \\
\hline LEH-18-R & $\begin{array}{l}\text { TCGACACGTTCGGTAAACACAAGTCCTTTGCACGAGCAGATATGGAACACGCAC } \\
\text { ACCGCATCATAGCTCATGACTTTGAACAGGCCAGCCA }\end{array}$ \\
\hline LEH-19-R & Same as LEH-11-R \\
\hline LEH-20-F & Same as LEH-5-F \\
\hline LEH-21-F & Same as LEH-13-F \\
\hline LEH-21-R & Same as LEH-13-R \\
\hline LEH-22-F & $\begin{array}{l}\text { AAACTCTGGCTGGCCTTTTCAAAGTCGTGAGCGTGGATGCGGTGTGCGTGTTCC } \\
\text { ATATCTGCTCGTGCAAAGGACTTGTGTTTACCGAACGTGTCGA }\end{array}$ \\
\hline LEH-22-R & TCGACACGTTCGGTAAACACAAGTCCTTTGCACGAGCAGATATGGAACACGCAC \\
\hline LEH-23-R & Same as LEH-16-R \\
\hline
\end{tabular}

Table S6. List of primers for LEH libraries A, B, C and D.

\begin{tabular}{|c|c|c|c|}
\hline Library & $\begin{array}{c}\text { Primer } \\
\text { mix }\end{array}$ & Name & Sequence ( $5^{\prime}$ to $\left.3^{\prime}\right)$ \\
\hline & & $\mathrm{LEH}-15(\mathrm{~S}+\mathrm{P})+19(\mathrm{P}+\mathrm{A})-\mathrm{F}$ & $\begin{array}{l}\text { GGGCGTCCAAGGACYCAGCCGCCGGCSCAGCCTCGACTCC } \\
\text { GGACG }\end{array}$ \\
\hline & & $\mathrm{LEH}-15(\mathrm{~S}+\mathrm{P})+19(\mathrm{~K})-\mathrm{F}$ & $\begin{array}{l}\text { GGGCGTCCAAGGACYCAGCCGCCGGCAAAGCCTCGACTCC } \\
\text { GGACG }\end{array}$ \\
\hline & & LEH-15(S+P) +19(D)-F & $\begin{array}{l}\text { GGGCGTCCAAGGACYCAGCCGCCGGCGATGCCTCGACTCC } \\
\text { GGACG }\end{array}$ \\
\hline & & $\mathrm{LEH}-15(\mathrm{~K})+19(\mathrm{P}+\mathrm{A})-\mathrm{F}$ & $\begin{array}{l}\text { GGGCGTCCAAGGACAAAGCCGCCGGCSCAGCCTCGACTCC } \\
\text { GGACG }\end{array}$ \\
\hline & & LEH-15(K)+19(K)-F & $\begin{array}{l}\text { GGGCGTCCAAGGACAAAGCCGCCGGCAAAGCCTCGACTCC } \\
\text { GGACG }\end{array}$ \\
\hline
\end{tabular}




\begin{tabular}{|c|c|c|c|}
\hline Library & $\begin{array}{l}\text { Primer } \\
\text { mix }\end{array}$ & Name & Sequence ( 5 ' to $\left.3^{\prime}\right)$ \\
\hline \multirow[t]{10}{*}{ A } & $F_{1}$ & LEH-15(K) +19(D)-F & $\begin{array}{l}\text { GGGCGTCCAAGGACAAAGCCGCCGGCGATGCCTCGACTC } \\
\text { CGGACG }\end{array}$ \\
\hline & & LEH-15(D) $+19(\mathrm{P}+\mathrm{A})-\mathrm{F}$ & $\begin{array}{l}\text { GGGCGTCCAAGGACGATGCCGCCGGCSCAGCCTCGACTCC } \\
\text { GGACG }\end{array}$ \\
\hline & & LEH-15(D) +19(K)-F & $\begin{array}{l}\text { GGGCGTCCAAGGACGATGCCGCCGGCAAAGCCTCGACTC } \\
\text { CGGACG }\end{array}$ \\
\hline & & LEH-15(D) +19(D)-F & $\begin{array}{l}\text { GGGCGTCCAAGGACGATGCCGCCGGCGATGCCTCGACTC } \\
\text { CGGACG }\end{array}$ \\
\hline & \multirow{6}{*}{ Ri } & LEH-74(LVF)+78(VF)-R & $\begin{array}{l}\text { ACCGCATCGATGCTGAMGACGGTGAAGAVGCCAGCCAGA } \\
\text { GTTTGC }\end{array}$ \\
\hline & & LEH-74(LVF)+78(Y)-R & $\begin{array}{l}\text { ACCGCATCGATGCTATAGACGGTGAAGAVGCCAGCCAGA } \\
\text { GTTTGC }\end{array}$ \\
\hline & & LEH-74(LVF)+78(M)-R & $\begin{array}{l}\text { ACCGCATCGATGCTCATGACGGTGAAGAVGCCAGCCAGA } \\
\text { GTTTGC }\end{array}$ \\
\hline & & LEH-74(Y)+78(VF)-R & $\begin{array}{l}\text { ACCGCATCGATGCTGAMGACGGTGAAATAGCCAGCCAGA } \\
\text { GTTTGC }\end{array}$ \\
\hline & & LEH-74(Y)+78(Y)-R & $\begin{array}{l}\text { ACCGCATCGATGCTATAGACGGTGAAATAGCCAGCCAGAG } \\
\text { TTTGC }\end{array}$ \\
\hline & & LEH-74(Y)+78(M)-R & $\begin{array}{l}\text { ACCGCATCGATGCTCATGACGGTGAAATAGCCAGCCAGAG } \\
\text { TTTGC }\end{array}$ \\
\hline & & LEH-76(TP) $+85(\mathrm{TP})-\mathrm{F}$ & $\begin{array}{l}\text { CTGGCTGGCCTGTTCMCAGTCATGAGCATCGATGCGGTG } \\
\text { GAGMCATTCCATATCGGCTCG }\end{array}$ \\
\hline & & LEH-76(TP) $+85(\mathrm{~K})-\mathrm{F}$ & $\begin{array}{l}\text { CTGGCTGGCCTGTTCMCAGTCATGAGCATCGATGCGGTG } \\
\text { GAGAAATTCCATATCGGCTCG }\end{array}$ \\
\hline & & LEH-76(TP) +85(D)-F & $\begin{array}{l}\text { CTGGCTGGCCTGTTCMCAGTCATGAGCATCGATGCGGTG } \\
\text { GAGGATTTCCATATCGGCTCG }\end{array}$ \\
\hline & & LEH-76(K) $+85(\mathrm{TP})-\mathrm{F}$ & $\begin{array}{l}\text { CTGGCTGGCCTGTTCAAAGTCATGAGCATCGATGCGGTGG } \\
\text { AGMCATTCCATATCGGCTCG }\end{array}$ \\
\hline & $\mathrm{F}_{2}$ & $\mathrm{LEH}-76(\mathrm{~K})+85(\mathrm{~K})-\mathrm{F}$ & $\begin{array}{l}\text { CTGGCTGGCCTGTTCAAAGTCATGAGCATCGATGCGGTGG } \\
\text { AGAAATTCCATATCGGCTCG }\end{array}$ \\
\hline
\end{tabular}




\begin{tabular}{|c|c|c|c|}
\hline Library & $\begin{array}{c}\text { Primer } \\
\text { mix }\end{array}$ & Name & Sequence $\left(5^{\prime}\right.$ to $\left.3^{\prime}\right)$ \\
\hline \multirow{8}{*}{ B } & & $\mathrm{LEH}-76(\mathrm{~K})+85(\mathrm{D})-\mathrm{F}$ & $\begin{array}{l}\text { CTGGCTGGCCTGTTCAAAGTCATGAGCATCGATGCGGTGG } \\
\text { AGGATTTCCATATCGGCTCG }\end{array}$ \\
\hline & & $\mathrm{LEH}-76(\mathrm{D})+85(\mathrm{TP})-\mathrm{F}$ & $\begin{array}{l}\text { CTGGCTGGCCTGTTCGATGTCATGAGCATCGATGCGGTGG } \\
\text { AGMCATTCCATATCGGCTCG }\end{array}$ \\
\hline & & LEH-76(D) $+85(\mathrm{~K})-\mathrm{F}$ & $\begin{array}{l}\text { CTGGCTGGCCTGTTCGATGTCATGAGCATCGATGCGGTGG } \\
\text { AGAAATTCCATATCGGCTCG }\end{array}$ \\
\hline & & LEH-76(D) +85(D)-F & $\begin{array}{l}\text { CTGGCTGGCCTGTTCGATGTCATGAGCATCGATGCGGTGG } \\
\text { AGGATTTCCATATCGGCTCG }\end{array}$ \\
\hline & & $\begin{array}{l}\text { LEH-114(LVF) +116(IVF)- } \\
\text { R }\end{array}$ & TGGAAGACTCCGAGGAHTGAGAVGTTGTAGCTCTTGCCG \\
\hline & \multirow{3}{*}{$\mathrm{R}_{2}$} & LEH-114 (LVF) $+116(Y)-R$ & TGGAAGACTCCGAGATATGAGAVGTTGTAGCTCTTGCCG \\
\hline & & LEH-114(Y) +116(IVF)-R & TGGAAGACTCCGAGGAHTGAATAGTTGTAGCTCTTGCCG \\
\hline & & $\mathrm{LEH}-114(\mathrm{Y})+116(\mathrm{Y})-\mathrm{R}$ & TGGAAGACTCCGAGATATGAATAGTTGTAGCTCTTGCCG \\
\hline & \multirow{8}{*}{$\mathrm{F}_{3}$} & LEH-92(KN) +96(DY)-F & $\begin{array}{l}\text { CCATATCGGCTCGAGTAAKGGACTTGTGKACACCGAACGT } \\
\text { GTCGATG }\end{array}$ \\
\hline & & LEH-92(KN) +96(K)-F & $\begin{array}{l}\text { CCATATCGGCTCGAGTAAKGGACTTGTGAAAACCGAACGT } \\
\text { GTCGATG }\end{array}$ \\
\hline & & LEH-92(KN)+96(P)-F & $\begin{array}{l}\text { CCATATCGGCTCGAGTAAKGGACTTGTGCCGACCGAACGT } \\
\text { GTCGATG }\end{array}$ \\
\hline & & LEH-92(D) +96(DY)-F & $\begin{array}{l}\text { CCATATCGGCTCGAGTGATGGACTTGTGKACACCGAACGT } \\
\text { GTCGATG }\end{array}$ \\
\hline & & LEH-92(D) +96(K)-F & $\begin{array}{l}\text { CCATATCGGCTCGAGTGATGGACTTGTGAAAACCGAACGT } \\
\text { GTCGATG }\end{array}$ \\
\hline & & LEH-92(D) +96(P)-F & $\begin{array}{l}\text { CCATATCGGCTCGAGTGATGGACTTGTGCCGACCGAACGT } \\
\text { GTCGATG }\end{array}$ \\
\hline & & LEH-92(P) +96(DY)-F & $\begin{array}{l}\text { CCATATCGGCTCGAGTCCGGGACTTGTGKACACCGAACGT } \\
\text { GTCGATG }\end{array}$ \\
\hline C & & LEH-92(P) +96(K)-F & $\begin{array}{l}\text { CCATATCGGCTCGAGTCCGGGACTTGTGAAAACCGAACGT } \\
\text { GTCGATG }\end{array}$ \\
\hline
\end{tabular}




\begin{tabular}{|c|c|c|c|}
\hline Library & $\begin{array}{l}\text { Primer } \\
\text { mix }\end{array}$ & Name & Sequence $\left(5^{\prime}\right.$ to $\left.3^{\prime}\right)$ \\
\hline & & LEH-92(P) +96(P)-F & $\begin{array}{l}\text { CCATATCGGCTCGAGTCCGGGACTTGTGCCGACCGAACGT } \\
\text { GTCGATG }\end{array}$ \\
\hline & \multirow{4}{*}{$\mathrm{R}_{3}$} & $\begin{array}{l}\text { LEH-139(VF) +147(LVF)- } \\
\text { R }\end{array}$ & $\begin{array}{l}\text { TTAGCCGCGGAVGGGAAGGTCGACAGCTTCTTCGAMTTC } \\
\text { GCGCAGATCGAAGT }\end{array}$ \\
\hline & & LEH-139(VF) +147(Y)-R & $\begin{array}{l}\text { TTAGCCGCGATAGGGAAGGTCGACAGCTTCTTCGAMTTC } \\
\text { GCGCAGATCGAAGT }\end{array}$ \\
\hline & & LEH-139(Y) +147(LVF)-R & $\begin{array}{l}\text { TTAGCCGCGGAVGGGAAGGTCGACAGCTTCTTCATATTCG } \\
\text { CGCAGATCGAAGT }\end{array}$ \\
\hline & & LEH-139(Y) +147(Y)-R & $\begin{array}{l}\text { TTAGCCGCGATAGGGAAGGTCGACAGCTTCTTCATATTCG } \\
\text { CGCAGATCGAAGT }\end{array}$ \\
\hline \multirow{10}{*}{ D } & \multirow{9}{*}{$\mathrm{F}_{4}$} & $\mathrm{LEH}-32(\mathrm{VF})+45(\mathrm{ED})-\mathrm{F}$ & $\begin{array}{l}\text { CGTTCTGGAGTTCKTCGACGCACTGACCAGTAATGATGCT } \\
\text { GCAAAACTCATTGAKTACTTTGCAGAAGACA }\end{array}$ \\
\hline & & $\mathrm{LEH}-32(\mathrm{VF})+45(\mathrm{~K})-\mathrm{F}$ & $\begin{array}{l}\text { CGTTCTGGAGTTCKTCGACGCACTGACCAGTAATGATGCT } \\
\text { GCAAAACTCATTAAATACTTTGCAGAAGACA }\end{array}$ \\
\hline & & $\mathrm{LEH}-32(\mathrm{VF})+45(\mathrm{P})-\mathrm{F}$ & $\begin{array}{l}\text { CGTTCTGGAGTTCKTCGACGCACTGACCAGTAATGATGCT } \\
\text { GCAAAACTCATTCCGTACTTTGCAGAAGACA }\end{array}$ \\
\hline & & $\mathrm{LEH}-32(\mathrm{Y})+45(\mathrm{ED})-\mathrm{F}$ & $\begin{array}{l}\text { CGTTCTGGAGTTCTATGACGCACTGACCAGTAATGATGCT } \\
\text { GCAAAACTCATTGAKTACTTTGCAGAAGACA }\end{array}$ \\
\hline & & LEH-32(Y) +45(K)-F & $\begin{array}{l}\text { CGTTCTGGAGTTCTATGACGCACTGACCAGTAATGATGCT } \\
\text { GCAAAACTCATTAAATACTTTGCAGAAGACA }\end{array}$ \\
\hline & & $\mathrm{LEH}-32(\mathrm{Y})+45(\mathrm{P})-\mathrm{F}$ & $\begin{array}{l}\text { CGTTCTGGAGTTCTATGACGCACTGACCAGTAATGATGCT } \\
\text { GCAAAACTCATTCCGTACTTTGCAGAAGACA }\end{array}$ \\
\hline & & $\mathrm{LEH}-32(\mathrm{M})+45(\mathrm{ED})-\mathrm{F}$ & $\begin{array}{l}\text { CGTTCTGGAGTTCATGGACGCACTGACCAGTAATGATGCT } \\
\text { GCAAAACTCATTGAKTACTTTGCAGAAGACA }\end{array}$ \\
\hline & & $\mathrm{LEH}-32(\mathrm{M})+45(\mathrm{~K})-\mathrm{F}$ & $\begin{array}{l}\text { CGTTCTGGAGTTCATGGACGCACTGACCAGTAATGATGCT } \\
\text { GCAAAACTCATTAAATACTTTGCAGAAGACA }\end{array}$ \\
\hline & & $\mathrm{LEH}-32(\mathrm{M})+45(\mathrm{P})-\mathrm{F}$ & $\begin{array}{l}\text { CGTTCTGGAGTTCATGGACGCACTGACCAGTAATGATGCT } \\
\text { GCAAAACTCATTCCGTACTTTGCAGAAGACA }\end{array}$ \\
\hline & & LEH-8o(IVF) -R & AACGTCTCCACCGCATCGAHGCTCATGACGGTGAAC \\
\hline
\end{tabular}




\begin{tabular}{|c|c|c|c|}
\hline Library & $\begin{array}{c}\text { Primer } \\
\text { mix }\end{array}$ & Name & Sequence (5' to $\left.3^{\prime}\right)$ \\
\hline & $\mathrm{R}_{4}$ & LEH-8o(Y) -R & AACGTCTCCACCGCATCATAGCTCATGACGGTGAAC \\
\hline & \multirow{2}{*}{$\mathrm{F}_{5}$} & LEH-8o(IVF) -F & GTTCACCGTCATGAGCDTCGATGCGGTGGAGACGTT \\
\hline & & LEH-8o(Y) -F & GTTCACCGTCATGAGCTATGATGCGGTGGAGACGTT \\
\hline & \multirow{3}{*}{$R_{5}$} & LEH-124(KE) -R & CCCGTAATCTTGCCTTYGGTGAGCTGGAAGACT \\
\hline & & LEH-124(P) -R & CCCGTAATCTTGCCCGGGGTGAGCTGGAAGACT \\
\hline & & LEH-124(D) -R & CCCGTAATCTTGCCATCGGTGAGCTGGAAGACT \\
\hline
\end{tabular}

Table S7. List of primers for ISM of LEH.

\begin{tabular}{|c|c|c|c|}
\hline Library & $\begin{array}{c}\text { Primer } \\
\text { mix }\end{array}$ & Name & Sequence (5' to 3 ') \\
\hline \multirow{2}{*}{$\begin{array}{l}A-C(A- \\
5-C_{1} \\
\text { visit C) }\end{array}$} & $\mathrm{F}_{3}$ & - & - \\
\hline & $\mathrm{R}_{3}$ & - & - \\
\hline \multirow{2}{*}{$\begin{array}{c}\text { B-C (B- } \\
\text { 1-F12 } \\
\text { visit C) }\end{array}$} & $\mathrm{F}_{3}$ & - & - \\
\hline & $\mathrm{R}_{3}$ & - & - \\
\hline & & $\begin{array}{l}\mathrm{E}-5-\mathrm{A} 8-76(\mathrm{TP})+85(\mathrm{TP})- \\
\mathrm{F}\end{array}$ & $\begin{array}{l}\text { CTGGCTGGCCTCTTCMCAGTCTTCAGCATCGATGCGGTGG } \\
\text { AGMCATTCCATATCGGCTCG }\end{array}$ \\
\hline & & E-5-A8-76(TP) $+85(\mathrm{~K})-\mathrm{F}$ & $\begin{array}{l}\text { CTGGCTGGCCTCTTCMCAGTCTTCAGCATCGATGCGGTGG } \\
\text { AGAAATTCCATATCGGCTCG }\end{array}$ \\
\hline & & E-5-A8-76(TP) +85(D)-F & $\begin{array}{l}\text { CTGGCTGGCCTCTTCMCAGTCTTCAGCATCGATGCGGTGG } \\
\text { AGGATTTCCATATCGGCTCG }\end{array}$ \\
\hline & & E-5-A8-76(K) +85(TP)-F & $\begin{array}{l}\text { CTGGCTGGCCTCTTCAAAGTCTTCAGCATCGATGCGGTGG } \\
\text { AGMCATTCCATATCGGCTCG }\end{array}$ \\
\hline
\end{tabular}




\begin{tabular}{|c|c|c|c|}
\hline Library & $\begin{array}{l}\text { Primer } \\
\text { mix }\end{array}$ & Name & Sequence ( 5 ' to $\left.3^{\prime}\right)$ \\
\hline \multirow{6}{*}{$\begin{array}{l}\text { A-C-B } \\
(\text { E-5-A8 } \\
\text { visit B) }\end{array}$} & \multirow{5}{*}{ F2' } & $\mathrm{E}-5-\mathrm{A} 8-76(\mathrm{~K})+85(\mathrm{~K})-\mathrm{F}$ & $\begin{array}{l}\text { CTGGCTGGCCTCTTCAAAGTCTTCAGCATCGATGCGGTGG } \\
\text { AGAAATTCCATATCGGCTCG }\end{array}$ \\
\hline & & E-5-A8-76(K)+85(D)-F & $\begin{array}{l}\text { CTGGCTGGCCTCTTCAAAGTCTTCAGCATCGATGCGGTGG } \\
\text { AGGATTTCCATATCGGCTCG }\end{array}$ \\
\hline & & E-5-A8-76(D) +85(TP)-F & $\begin{array}{l}\text { CTGGCTGGCCTCTTCGATGTCTTCAGCATCGATGCGGTGG } \\
\text { AGMCATTCCATATCGGCTCG }\end{array}$ \\
\hline & & E-5-A8-76(D) +85(K)-F & $\begin{array}{l}\text { CTGGCTGGCCTCTTCGATGTCTTCAGCATCGATGCGGTGG } \\
\text { AGAAATTCCATATCGGCTCG }\end{array}$ \\
\hline & & E-5-A8-76(D) +85(D)-F & $\begin{array}{l}\text { CTGGCTGGCCTCTTCGATGTCTTCAGCATCGATGCGGTGG } \\
\text { AGGATTTCCATATCGGCTCG }\end{array}$ \\
\hline & $\mathbf{R}_{2}$ & - & - \\
\hline \multirow{7}{*}{$\begin{array}{l}\text { B-C-A } \\
(\text { F-6-A9 } \\
\text { visit A) }\end{array}$} & $\mathrm{F}_{1}$ & - & - \\
\hline & \multirow{6}{*}{$\mathrm{Ri}^{\prime}$} & $\begin{array}{l}\text { F-6-A9- } \\
74(\mathrm{LVF})+78(\mathrm{VF})-\mathrm{R}\end{array}$ & $\begin{array}{l}\text { ACCGCATCGATGCTGAMGACTTTGAAGAVGCCAGCCAGA } \\
\text { GTTTGC }\end{array}$ \\
\hline & & $\begin{array}{l}\text { F-6-A9-74(LVF)+78(Y)- } \\
\text { R }\end{array}$ & $\begin{array}{l}\text { ACCGCATCGATGCTATAGACTTTGAAGAVGCCAGCCAGAG } \\
\text { TTTGC }\end{array}$ \\
\hline & & $\begin{array}{l}\text { F-6-A9-74(LVF)+78(M)- } \\
\text { R }\end{array}$ & $\begin{array}{l}\text { ACCGCATCGATGCTCATGACTTTGAAGAVGCCAGCCAGAG } \\
\text { TTTGC }\end{array}$ \\
\hline & & $\mathrm{F}-6-\mathrm{A} 9-74(\mathrm{Y})+78(\mathrm{VF})-\mathrm{R}$ & $\begin{array}{l}\text { ACCGCATCGATGCTGAMGACTTTGAAATAGCCAGCCAGA } \\
\text { GTTTGC }\end{array}$ \\
\hline & & $\mathrm{F}-6-\mathrm{A} 9-74(\mathrm{Y})+78(\mathrm{Y})-\mathrm{R}$ & $\begin{array}{l}\text { ACCGCATCGATGCTATAGACTTTGAAATAGCCAGCCAGAG } \\
\text { TTTGC }\end{array}$ \\
\hline & & F-6-A9- $74(\mathrm{Y})+78(\mathrm{M})-\mathrm{R}$ & $\begin{array}{l}\text { ACCGCATCGATGCTCATGACTTTGAAATAGCCAGCCAGAG } \\
\text { TTTGC }\end{array}$ \\
\hline \multirow{5}{*}{$\begin{array}{c}\text { A-C-B- } \\
\text { D (I-4- } \\
\text { H1o } \\
\text { visit D) }\end{array}$} & $\mathrm{F}_{4}$ & - & - \\
\hline & \multirow[t]{2}{*}{$\mathrm{R}_{4}^{\prime}$} & $\mathrm{I}-4-\mathrm{H} 10-8 \mathrm{o}(\mathrm{IVF})-\mathrm{R}$ & АTTTCTCCACCGCATCGAHGCTGAAGACATCGAAG \\
\hline & & $\mathrm{I}-4-\mathrm{H} 10-8 \mathrm{o}(\mathrm{Y})-\mathrm{R}$ & ATTTCTCCACCGCATCATAGCTGAAGACATCGAA \\
\hline & \multirow[b]{2}{*}{$\mathrm{F}_{5}$} & $\mathrm{I}-4-\mathrm{H} 10-8 \mathrm{o}(\mathrm{IVF})-\mathrm{F}$ & CTTCGATGTCTTCAGCDTCGATGCGGTGGAGAAAT \\
\hline & & $\mathrm{I}-4-\mathrm{H}_{10}-8 \mathrm{o}(\mathrm{Y})-\mathrm{F}$ & CTTCGATGTCTTCAGCTATGATGCGGTGGAGAAAT \\
\hline
\end{tabular}




\begin{tabular}{|c|c|c|c|}
\hline Library & $\begin{array}{c}\text { Primer } \\
\text { mix }\end{array}$ & Name & Sequence (5' to $\left.3^{\prime}\right)$ \\
\hline & $\mathrm{R}_{5}$ & - & - \\
\hline \multirow{6}{*}{$\begin{array}{c}\text { B-C-A- } \\
\text { D (G-7- } \\
\mathrm{H}_{2} \\
\text { visit D })\end{array}$} & $\mathrm{F}_{4}$ & - & - \\
\hline & \multirow[b]{2}{*}{$\mathrm{R}_{4} "$} & G7-H2-LEH-8o(IVF) -R & $\begin{array}{l}\text { ATGTCTCCACCGCATCGAHGCTGAAGACTTTGAAG } \\
\end{array}$ \\
\hline & & $\mathrm{G}_{7}-\mathrm{H}_{2}-\mathrm{LEH}-8 \mathrm{o}(\mathrm{Y})-\mathrm{R}$ & ATGTCTCCACCGCATCATAGCTGAAGACTTTGAAG \\
\hline & \multirow[t]{2}{*}{ F5" } & G7-H2-LEH-8o(IVF) -F & CTTCAAAGTCTTCAGCDTCGATGCGGTGGAGACAT \\
\hline & & $\mathrm{G}_{7}-\mathrm{H}_{2}-\mathrm{LEH}-8 \mathrm{o}(\mathrm{Y})-\mathrm{F}$ & CTTCAAAGTCTTCAGCTATGATGCGGTGGAGACAT \\
\hline & $\mathrm{R}_{5}$ & - & - \\
\hline
\end{tabular}

Table S8. Analytic conditions of GC. ${ }^{\dagger}$

\begin{tabular}{|c|c|c|c|c|}
\hline Entry & Diol & Procedure & Retention time & Comment \\
\hline $\mathbf{1}$ & $\begin{array}{l}(S, S) \mathbf{- 2} \\
(R, R)-\mathbf{2}\end{array}$ & $\begin{array}{l}130{ }^{\circ} \mathrm{C}, 2.5^{\circ} \mathrm{C} / \mathrm{min}, 135^{\circ} \mathrm{C}, 50^{\circ} \mathrm{C} / \mathrm{min}, \\
200{ }^{\circ} \mathrm{C} . \mathrm{H}_{2}: 2.7 \text { bar }\end{array}$ & $\begin{array}{l}(\mathrm{S}, \mathrm{S})-2: 1.7 \mathrm{~min} \\
(R, R)-2: 1.9 \mathrm{~min}\end{array}$ & $\begin{array}{l}\text { for ee } \\
\text { screening }\end{array}$ \\
\hline 2 & $\begin{array}{l}(S, S) \mathbf{- 2} \\
(R, R)-\mathbf{2}\end{array}$ & $\begin{array}{l}80{ }^{\circ} \mathrm{C}(4 \mathrm{~min}), 5{ }^{\circ} \mathrm{C} / \mathrm{min}, 130{ }^{\circ} \mathrm{C}(2 \mathrm{~min}) \\
50{ }^{\circ} \mathrm{C} / \mathrm{min}, 200{ }^{\circ} \mathrm{C}(1 \mathrm{~min}) . \mathrm{H}_{2}: 1.5 \mathrm{bar}\end{array}$ & $\begin{array}{l}\text { epoxide 1: } 5.6 \mathrm{~min} \\
(S, S)-2: 13.8 \mathrm{~min} \\
(R, R)-2: 14.2 \mathrm{~min}\end{array}$ & $\begin{array}{l}\text { for ee and } \\
\text { conversion }\end{array}$ \\
\hline
\end{tabular}

${ }^{\dagger} \mathrm{GC}$ column is Hydrodex- $\beta$-TBDAc, $25 \mathrm{~m} \times 0.25 \mathrm{~mm}$ ID, each entry was identified based on authentic racemic mixture and enantiomer product.

${ }^{\ddagger}$ The product absolute configuration was established by chiral Gas Chromatography (GC) analysis according to the elution order. $(R)$ enantiomer was eluted before the $(S)$ enantiomer.40 